\title{
SALUD MENTAL Y MALESTAR EMOCIONAL EN PACIENTES CON CÁNCER
}

\author{
MENTAL HEALTH AND DISTRESS IN CANCER PATIENTS
}

Manuel Hernández', Juan Antonio Cruzado², Carmen Prado 3 , Emilio Rodríguez 4 , Cristina Hernández', Miguel Ángel González ${ }^{1,6}$, Juan Carlos Martín ${ }^{5,7}$

' Unidad de Psicooncología. Servicio de Psiquiatría. Hospital Universitario de Basurto. Bilbao. Osakidetza.

2 Facultad de Psicología. Universidad Complutense de Madrid.

${ }^{3}$ Servicio de Cirugía Pediátrica. Hospital Universitario de Cruces. Baracaldo. Osakidetza.

${ }^{4}$ Unidad de Comunicación Médica. Osakidetza.

${ }^{5}$ Servicio de Radioterapia Oncológica. Hospital Universitario de Basurto. Bilbao. Osakidetza.

${ }^{6}$ Departamento de Neurociencias. Universidad del País Vasco.

7 Departamento de Radiología y Medicina Física. Universidad del País Vasco.

Resumen

Objetivo: Conocer los problemas de salud mental y de malestar psíquico de los pacientes con cáncer, las barreras que pueden incidir en su escasa detección y en la falta de atención psicológica específica a los pacientes que la precisan, así como las vías que clínicos e investigadores están desarrollando para solventarlas. Un conocimiento real de estos problemas facilitaría el diseño, implementación o mejora de los recursos asistenciales, para el paciente oncológico.

Método: Revisión bibliográfica de las investigaciones sobre el tema en las últimas décadas.

Resultados: Los pacientes con cáncer presentan elevada prevalencia de trastornos psicopatológicos y síntomas de ansiedad, depresión y malestar emocional (distress), en distintos momentos de la enfermedad, relacionados con diversos factores médicos, físicos, psicológicos y sociales. Los datos no permiten concluir sobre la prevalencia real puntual de dichos trastornos y síntomas, debido a las diferentes metodologías de investigación, y menos aun sobre su evolución durante la enfermedad, y
Abstract

Purpose: Identify mental health problems and distress in cancer patients; recognise the obstacles to their detection and the lack of specific psychological care for patients who need it; and determine the pathways that are being developed to address them. A true understanding of these problems would facilitate the design, implementation and improvement of health-care resources for oncologic patients.

Methods: Literature review of the investigations on the subject from the last decades.

Results: During their illness, cancer patients show a high prevalence of psychopathological disorders, anxiety symptoms, depression and distress, related to several medical, physical, psychological and social factors. To discover the protective and predictive factors of worsening, data analysis does not indicate the real punctual prevalence of these disorders and symptoms -mainly due to the different investigation methods used-, neither does it show the evolution during the illness and afterwards.

\section{Correspondencia:}

Manuel Hernández Blázquez, PhD

Coordinador Unidad de Psicooncología

Hospital Universitario de Basurto. Osakidetza.

Avda. Montevideo, 18.

48013. Bilbao. España.

E-mail: m.pernetas@cop.es 
en momentos posteriores a la misma, para conocer los posibles factores predictivos de empeoramiento o protección.

Diversas barreras dificultan una adecuada evaluación psicológica a los pacientes con cáncer, lo que conlleva una falta de atención específica y especializada cuando se precisa, un empeoramiento clínico de esos pacientes, y dificultades de adherencia a los tratamientos médicos. Las vías de solución favorecidas en la última década son la formación a los profesionales sanitarios en herramientas de comunicación y el desarrollo de herramientas de detección de trastornos mentales y malestar emocional adecuadas a la realidad clínica de los pacientes, con resultados dispares.

Conclusiones: Es preciso seguir mejorando los sistemas de detección de problemas de salud mental y de malestar emocional en pacientes oncológicos, para darles la atención psicológica específica cuando se precise. Un conocimiento real de estos problemas favorecerá el diseño de programas asistenciales especializados en psicooncología y psicología clínica. Es necesaria la coordinación y accesibilidad del psicólogo en los equipos médicos.

Palabras claves: Salud mental, malestar emocional, detección, asistencia psicológica, cáncer, psicooncología.
Several barriers make psychological assessment of cancer patients difficult, this leads to a lack of specific and specialised attention when needed, a clinic worsening of these patients and difficulty in adhesion to medical treatments.

Training in communication tools for sanitary professionals and the development of distress and mental disorder detection tools, according to the clinic reality of the patients, are the solutions preferred in this last decade, though with irregular results.

Conclusions: In order to offer cancer patients a specific psychological attention when needed, mental health problems and distress detection tools need to keep improving. A real knowledge of these problems will result in the design of specialised psycho-oncological and clinic psychological assistance programmes. To guarantee access and coordination with the psychologist in medical teams is essential.

Keywords: Mental health, distress, screening, psychological assistance, cancer, psycho-oncology.

\section{INTRODUCCIÓN}

La cuestión de las necesidades y problemas psicológicos en personas que sufren un cáncer, ocupa el interés de múltiples estudios. Nos han interesado los que estudian los efectos que la enfermedad acarrea desde el punto de vista emocional, personal, familiar, social, etc. $Y$ en particular los que indagan sobre los problemas de salud mental del paciente, y sus reacciones sintomáticas de ansiedad, depresión y malestar psíquico.

Se llevó a cabo una revisión bibliográfica ${ }^{(1)}$ consultando principalmente las bases de datos siguientes: 1) Books@Ovid (hasta marzo 2012; 2) Journals@Ovid; 3) Ovid MEDLINE(R) (1950-marzo 2012); 4) PAS-
CAL (1984 -marzo 2012); 5) PsycARTICLES Full Text; 6) PsycBOOKS (1806 - marzo 2012); 7) PsycINFO (1806 - marzo 2012); y 8) Revistas texto completo que subscribe Osakidetza. En todas ellas se han combinado los términos-clave "cancer", "chemotherapy", "radiation therapy", "radiotherapy", "psych\$", "adjust\$ disorder\$", "depressi $\$$ ", "anxiety", "emotion $\$$ ", "distress", "quality of life".

A partir de esos datos, indagamos sobre las dificultades que se presentan a la hora de realizar una adecuada detección y evaluación psicológica de estos pacientes, las barreras que se reconocen en la literatura sobre el tema, y los estudios que buscan cómo mejorar la situación. 


\section{TRASTORNOS PSICOPATOLÓGICOS Y SINTOMAS DE ANSIEDAD, DEPRESIÓN Y MALESTAR EMOCIONAL}

\section{Trastornos psicopatológicos}

Una reciente revisión ${ }^{(1)}$ muestra la importante presencia de trastornos psicopatológicos y síntomas de depresión, ansiedad y malestar emocional en pacientes con cáncer; más prevalentes que en la población general; sin embargo, los datos actuales no permiten establecer una prevalencia clara, debido a las diferentes metodologías de los estudios revisados, lo que influyen en la gran variabilidad de los resultados.

Así, por ejemplo, se informa de la frecuencia de trastornos adaptativos entre el 32\% del clásico estudio del PSYCOG (Psychosocial Collaborative Oncology Group $)^{(2)}$, y el $55 \%$ del estudio realizado en nuestro medio ${ }^{(3)}$. Estos trastornos varían en la población general, entre un 5 y 20\% como recoge el DSM-IV-TR ${ }^{(4)}$ (American Psychiatric Association, 2002)

Los trastornos del estado del animo observados oscilan desde el $6 \%$, ${ }^{(2)}$, o el $2,25-5 \%$ en nuestro medio ${ }^{(5,3)}$, hasta el 38 $50 \%$ que destacan algunas revisiones ${ }^{(6,7)}$. Mientras que su prevalencia anual en la población general española es del $4,4 \%{ }^{(8)}$.

El riesgo de suicidio, o la simple ideación autolítica, una clínica frecuentemente asociada la depresión ${ }^{(9)}$, supone el $9 \%$ de los casos que acude a la consulta de psiquiatría ${ }^{(10)}$, y varia entre el $1 \%$ al $20 \%$ según la gravedad y lo avanzado de la enfermedad $^{(11,12)}$; muy superior al $4,4 \%$ de ideas de suicidio y al $1 \%$ de riesgo real referidos en la población general española ${ }^{(13)}$.

Los trastornos de ansiedad varían igualmente, entre el $2,25 \%$ y el $7,8 \%$ en nuestro medio ${ }^{(5,3)}$, y entre el $2 \%$ y porcentajes del 16 al $44 \%$, en otros estudios y revisiones $^{(2,14,15)}$, mientras que la población general presenta estos trastornos en porcentajes entre el $2-6 \%{ }^{(16)}$.
La clínica psicopatológica y su evolución, en estos pacientes, se encuentra asociada o relacionada con factores de riesgo, algunos específicos de su situación de enfermedad y vital.

Así, para los trastornos adaptativos se ha descrito la influencia de la hospitalización ${ }^{(17)}$, el funcionamiento físico deteriorado, que influye en la sensación de ser una carga para los demás ${ }^{(18)}$, y la adaptación inicial a los tratamientos; juntos a factores subjetivos como la fortaleza/debilidad yoica, la percepción del apoyo social y la experiencia previa frente al estrés ${ }^{(19)}$.

Para los trastornos depresivos: el tener una edad más joven, y el género en ciertos subgrupos de pacientes, aunque no en todos los estudios ${ }^{(20-22)}$; el estadio avanzado de la enfermedad, quizás relacionado con la mayor limitación o la incapacidad física ${ }^{(23-25)}$; la presencia de síntomas físicos secuelas de los tratamientos ${ }^{(26)}$; y el tipo de enfermedad neoplásica, con mayor depresión en cáncer de páncreas, orofaríngeo y del sistema nervioso central| ${ }^{21,27)}$; e igualmente destacan la presencia de antecedentes psicológicos y trastornos previos ${ }^{(5)}$, el bajo apoyo social percibido ${ }^{(28)}$, y factores de riesgo biopsicosocial ${ }^{(22)}$.

Para la ideación/riesgo de suicidio, diversos estudios ${ }^{(9,11,29-31)}$ describen: el estadio avanzado y peor pronóstico de la enfermedad, el tipo y localización del tumor, mayor en cáncer de cabeza y cuello; la presencia de un trastorno de depresión, o la desesperanza, la falta de control y el sentimiento de desamparo; el incremento de síntomas físicos o su cronicidad $^{(32)}$; el Delirium y los estados confusionales; los antecedentes psicopatológicos, y la historia previa de suicidio.

$Y$ para los trastornos de ansiedad: una edad más joven, el ser mujer y tener una situación socioeconómica desfavorecida ${ }^{(16)}$; la adaptación psicológica premórbida con tendencias ansiosas, rasgos de personalidad obsesiva y dificultades personales, de 
pareja o laborales ${ }^{(33-36)}$; pero igualmente se destacan la situación avanzada de la enfermedad, el estado físico asociado y la situación de tratamiento ${ }^{(37-39)}$; la recidiva de la enfermedad ${ }^{(40)}$, factores orgánicos relacionados con la enfermedad, la toxicidad farmacológica, o el influjo directo de la enfermedad $^{(4,15)}$, y el dolor agudo no controlado $^{(2,15,41)}$.

\section{Síntomas de ansiedad, depresión y malestar emocional}

Respecto a los estudios sobre síntomas, cumplan o no criterio de trastorno psicopatológico, la prevalencia de síntomas de ansiedad y depresión es bastante alta. Pacientes en diferente situación de enfermedad, evolución y tratamiento, presentan síntomas depresivos entre un 17\% y un $25 \%$ en poblaciones españolas y europeas $^{(1,42)} ;$ y en otros países, entre un 2 y un $20 \%\left({ }^{(45,20)}\right.$.

Así mismo se observa un $24 \%$ de ansie$\operatorname{dad}^{(1,20,43)}$, que oscila entre $2 \%$ y un $44 \%$ en función del tipo de cáncer ${ }^{(15)}$; o entre un 15 y un $36 \%$ en poblaciones españolas y europeas ${ }^{(5,42,45)}$. Prevalencia que varía también en función de la herramienta de detección utilizada, entre un 25 y un $30 \%{ }^{(44)}$.

\section{Unas palabras sobre el concepto de Malestar emocional (distress) y su uso clínico}

En las últimas décadas, los estudios incluyen cada vez más este concepto de malestar psíquico; y su valoración en la clínica, según algunos autores, es fundamental:

"El malestar emocional se está convirtiendo en un elemento capital para valorar la salud y el bienestar de los pacientes con cáncer. Para los clínicos es el 'sexto signo vital', tan principal como la temperatura, la respiración, el ritmo cardiaco, la presión sanguínea o el dolor ${ }^{\prime \prime(46)}$.
Se trata de un concepto propuesto por la National Comprehensive Cancer Network ${ }^{(47)}$, para no estigmatizar ni cargar de contenido psicopatológico a las dificultades psicológicas de los pacientes con cáncer. Pero es impreciso, tanto respecto a los instrumentos utilizados para su evaluación operativa, como en su definición: "experiencia emocional desagradable que afecta al funcionamiento cognitivo, conductual, social, emocional y espiritual". Se propone la palabra "distress", para reflejar los distintos niveles de problemas psicológicos asociados al cáncer, que, lógicamente no todos son trastornos psicopatológicos ${ }^{(48)}$, pero la propia NCCN traduce esta palabra por "angustia"(49).

La imprecisión es más clara al considerar los instrumentos que usan distintos estudios para evaluar dicho malestar: por ejemplo, la escala K6, una encuesta de seis preguntas sobre el nivel de tristeza, nerviosismo, intranquilidad, desesperanza y falta de valor personal, validada con el DSM-IV ${ }^{(50)}$; el listado breve de síntomas, BSI-18 ${ }^{(51)}$, usado habitualmente para medir depresión, ansiedad y somatización; o incluso la puntuación total en la escala $\mathrm{HADS}^{(52)}$, argumentando que las subescalas de ansiedad y depresión, de que consta la misma, son poco específicas ${ }^{(53,54)}$. Es decir, que, en la práctica, estos estudios hablan del concepto de malestar psicológico a partir conceptos clínicos, como el de depresión y de ansiedad.

Difiere de ellos el Termómetro de malestar psíquico (DT -Distress Thermometer) desarrollado por Andrew Roth y colaboradores ${ }^{(55)}$, en asociación con una "Medida de detección y manejo del malestar psíquico" (DMSM). El malestar se objetiva de una forma simple mediante la imagen de un termómetro graduado de 0 a 10. Está apoyado por la $\mathrm{NCCN}^{(49)}$.

Quizás el mayor interés de este concepto radica en el intento de homologar, 
en las distintas instituciones asistenciales, un modo de detección rápida, que facilite la orientación a los distintos niveles de intervención, incluida la atención en salud mental.

Volviendo a los datos de prevalencia, también se encuentra una alta frecuencia de malestar emocional, en distintos momentos de la evolución de los pacientes, e igualmente con una gran variabilidad, que invalida en parte los datos. Se encuentra entre un $15 \%$ y un $33 \%$ de los casos, alcanzando al $63 \%$ si se toma a los pacientes que lo padecen en algún momento del estudio $^{(56)}$; y según el tipo de cáncer considerado, entre un $30 \%$ y un $44 \%{ }^{(57)}$, en concreto un $37 \%$ en el caso de pacientes en tratamiento de radioterapia ${ }^{(1)} ; \mathrm{y}$ alcanza niveles de intensidad que puede considerarse equivalente a un trastorno psicopatológico, entre el $41-47 \%$ de los $\operatorname{casos}^{(58)}$. Curiosamente, estos valores coinciden exactamente con la prevalencia de trastornos psiquiátricos totales del estudio del PSYCOG ${ }^{(2)}$.

En la población europea se describen moderados a altos niveles de malestar emocional en el 30\% de los casos -usando criterio HAD-T ${ }^{(42)}$.

Como factores de riesgo de malestar emocional se señalan: las secuelas de los tratamientos quirúrgicos en la esfera sexual y de la imagen corporal ${ }^{(59)}$, una edad menor, estar soltero, un nivel educativo inferior y dificultad para el acceso a la asistencia médica ${ }^{(60)} ;$ y complicaciones físicas post-tratamiento, y ciertos rasgos de personalidad $^{(56)}$.

\section{SITUACIÓN ACTUAL DE LA EVALUACIÓN Y ASISTENCIA PSICOLÓGICA}

\author{
Interés de la detección y evaluación \\ de los problemas psicopatológicos y \\ emocionales en los pacientes con cáncer
}

Se sabe que los problemas psicológicos pueden ser causa de complicaciones físicas, y afectar de modo adverso al estado general de la persona que los padece; es decir, un problema psicológico como la depresión es un riesgo añadido para la salud del paciente, quien disminuye o realiza con dificultad las actividades diarias de dieta, ejercicio y sueño, y puede incluso abandonar el tratamiento activo; un fenómeno recogido por el DSM-IV-TR [Código 316, y F54] $]^{(4)}$.

La presencia de problemas de salud mental hace que estos pacientes requieran más servicios médicos, tengan más dificultades a la hora de tomar decisiones, sean menos adherentes a sus tratamientos, y estén menos satisfechos con los cuidados médicos ${ }^{(61)}$, o genera pacientes híperdemandantes ${ }^{(62)}$.

Una adecuada y temprana detección aumentaría el número de pacientes que pueden beneficiarse de los recursos psicológicos ya disponibles en muchas instituciones, mejorando la adherencia al tratamiento médico, y dotando al paciente de estrategias de afrontamiento y de cambio, así como favorecer el tratamiento de sus posibles trastornos psicopatológicos o su sintomatología, para mejorar su evolución clínica, y la calidad de su vida ${ }^{(62-67)}$.

Inversamente, la falta de valoración de las necesidades psicológicas de los pacientes con cáncer, implica un mal uso de los recursos asistenciales; por ejemplo, que los sanitarios sólo envíen al paciente al psicólogo, o al psiquiatra, cuando ven que el paciente está severamente ansioso o deprimido, o aparecen ideas autolíticas, o cuando ya tiene un alto nivel malestar emocional y se manifiestan conflictos familiares, o bien, simplemente, cuando el cáncer está muy avanzado y el médico se ve en la difícil situación de comunicar a los pacientes que se han agotado los tratamientos activos $^{(3,68,69)}$. 


\section{Barreras o dificultades en la detección de problemas psicológicos}

Lamentablemente, a pesar de la alta prevalencia de trastornos psicopatológicos y de síntomas de ansiedad y depresión, y de ser el malestar emocional uno de los efectos más evidentes de tener un cáncer, la evaluación y asistencia específica a estos problemas y necesidades psicológicas, es deficitaria: sólo un $10 \%$ de pacientes con necesidad de ayuda psicológica la reciben $^{(58,70)}$, lo que se corresponde con lo señalado por la doctora Jimmie Holland, en su libro 'La cara humana del cáncer'(71) (p.124), que los pacientes con cáncer 'pueden pasear su clínica de angustia y depresión ante la mirada del médico oncólogo, sin que éste lo perciba, perdiéndose así la posibilidad de un asesoramiento

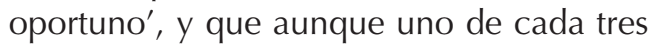
pacientes tratados en oncología está muy angustiado, sólo uno de cada diez recibe asesoramiento para obtener ayuda.

Son diversos los factores o barreras que pueden dificultar una detección adecuada.

\section{1) Unas debidas a lo propios pacientes:}

Algunos de ellos no informan de su malestar, o no piden ayuda, aunque puedan necesitarla; sólo el $36 \%$ de los pacientes con malestar emocional expresa el deseo de ser ayudado ${ }^{(72)}$, porque no se atreven a hablar de los problemas emocionales, o los niegan por el carácter estigmatizante que pueden tener ${ }^{(71,73-75)}$, o no encuentran la suficiente confianza o comodidad, en la relación con el médico ${ }^{(76)}$, o consideran que lo importante en las consultas es el tratamiento médico ${ }^{(6)}$. Estas razones pueden explicar el retraso de algunos pacientes a acudir al psicooncólogo, aun estando advertidos de dicho recurso por parte de los oncólogos. Jimmie Holland ha recogido diversas razones actitudinales, algunas achacables al miedo, el fatalismo, y ciertos estilos de personalidad, como la represión de la información inquietante, además de una pobre o nula relación médico-paciente, para explicar el retardo o las dificultades de los pacientes incluso para informar de sus síntomas físicos ${ }^{(77,78)}$.

\section{2) Otras debidas a los profesionales:}

Como dice irónicamente el radioterapeuta Rober Kagan, "el cáncer mata a menudo, la psique rara vez", haciendo una autocrítica a la actitud habitual del médico, que atiende a los problemas físicos del paciente pero deja de lado su situación emocional, considerándola secundaria. El $85 \%$ de las derivaciones del departamento de radioterapia a los profesionales sociosanitarios no las realizan los oncólogos, sino los técnicos que administran la radiación; quizás por su contacto más directo y diario con los pacientes ${ }^{(79)}$.

Las dificultades en los profesionales pueden obedecer a la falta de formación curricular en los temas psíquicos, o dificultades de carácter personal, como la propia angustia ante la situación vital de sus pacientes, o a actitudes nihilistas de etiquetar como 'normales' las reacciones psicológicas de los pacientes. Como dice David Spiegel ${ }^{(62,66)}$ : respuestas frecuentes en algunos sanitarios, como "es normal que esté deprimido, teniendo una enfermedad tan grave", pueden predisponer a una deficiente exploración de estos síntomas, a no tratar los posibles trastornos, y dar lugar a un aumento de las quejas somáticas, que obedecen al malestar emocional no tratado.

Lesley Fallowfield y sus colaboradores ${ }^{(80)}$, en un importante estudio sobre el tema, examinaban la capacidad de los médicos para detectar el estado emocional de sus pacientes, previamente evaluados con el cuestionario de salud general GHQ-12(81), resultando que los médicos 
sólo identificaban entre sus pacientes al $29 \%$ de los que habían sido previamente diagnosticados de alto nivel de malestar emocional.

En uno de los estudios dirigidos por Alex Mitchell sobre el tema ${ }^{(82)}$, concretaban las dificultades principales de los médicos para llevar a cabo una buena detección, en la falta de entrenamiento y de herramientas de detección adecuadas.

Las dificultades ligadas a la formación psicológica, y a la actitud del profesional han sido abordadas por distintos estu$\operatorname{dios}^{(80,82-85)}$, algunos resaltan la importancia de la ansiedad del evaluador, más que su nivel de formación ${ }^{(86)}$, o el uso de la negación como defensa psíquica ante las implicaciones emocionales que le suscitan sus pacientes ${ }^{(71)}$.

\section{3) Otras barreras se deben al contexto:}

Como la enorme presión asistencial bajo la que se trabaja, y el aumento de la demandas en las consultas médicas ${ }^{(71)}, \mathrm{o}$ la gravedad de la situación física en que se encuentran algunos pacientes, a la hora de ser evaluados, lo que dificulta el acceso a ellos, incluso la comunicación; la dificultad diagnóstica ante algunos síntomas, como la pérdida del apetito, la pérdida de peso, trastornos del sueño, fatiga/astenia, falta de energía, dificultad para concentrarse, enlentecimiento psicomotor, y otros, que pueden obedecer al contexto de la enfermedad o los tratamientos, pero que pueden corresponder también a un cuadro psicopatológico, de una depresión mayor, por ejemplo, lo que dificulta su reconocimiento y detección ${ }^{(87,88)}$.

Todo ello queda así mismo limitado por los problemas metodológicos de los estudios examinados, como la falta de un protocolo de investigación en este campo, de homogenización de los instrumentos utilizados, etc. También la escasez de estudios longitudinales impide conocer la evolución del malestar emocional y los trastornos psicopatológicos a lo largo del proceso del tratamiento oncológico.

\section{MÉTODOS PARA OPTIMIZAR LA EVALUACIÓN Y DETECCIÓN DEL MALESTAR EMOCIONAL Y FAVORECER LA DERIVACIÓN}

Existe un creciente interés por superar esas dificultades y barreras, aportando soluciones centradas en dos aspectos: la formación de los equipos sanitarios en psicopatología, y el desarrollo de instrumentos adecuados a la realidad asistencial de los pacientes, que les ayuden a detectar los problemas psicológicos, y se usen como criterio de derivación a los psicólogos.

\section{Formación del personal médico}

Según reflejan algunos estudios, la mayor parte de la morbilidad psiquiátrica de los pacientes con cáncer no se reconoce, y de ahí que no se trate $(58,70,71,80)$.

Para paliar este problema, algunos autores, como Steven Passik y sus colaboradores, han tratado de formar a los médicos oncólogos en el uso herramientas de detección para la depresión y síntomas asociados, y de calidad de vida, incluso facilitándoles el tratamiento de la depresión mediante un algoritmo de prescripción de medicación antidepresiva ${ }^{(89,90)}$. Subyace en ello la idea de que, si el equipo médico dispone de información sobre los problemas psicológicos, mejorará la derivación de los pacientes a los psicólogos ${ }^{(91)}$.

En este sentido, el Grupo de Estudio de Psicooncología del Sur de Europa (SEPOS), en distintos hospitales europeos (Italia, Portugal y España), ha desarrollado y aplicado programas para capacitar a los oncólogos en detección de problemas psicológicos, habilidades de comunicación y apoyo a los pacientes con cáncer y sus familiares ${ }^{(92,93)}$. Como muestra el trabajo de Luzia Travado, 
miembro de dicho grupo, tras evaluar a los oncólogos respecto a sus habilidades de comunicación, sus expectativas sobre los efectos de la comunicación, su orientación hacia los temas psicosociales, y la presencia de síntomas de agotamiento, se observa que aquéllos, en general, tienden a sentirse preparados técnicamente para la comunicación, excepto ante situaciones que viven como rechazo, también antes situaciones de incertidumbre, o a la hora detectar la ansiedad y la depresión, y de generar relaciones de confianza con la familia de los pacientes. Según este estudio, la habilidad para la comunicación depende de los conocimientos del médico en temas psicosociales, mientras que sus reacciones sintomáticas de cansancio, o astenia, se relacionan con una menor confianza en sus habilidades comunicativas y con la expectativa de resultados negativos.

Se considera que la formación a los profesionales debería mejorar sus herramientas de comunicación y consejo, para saber incluso aportar algunos cuidados emocionales al paciente, lo que respondería a una ética de mínimos en oncología, como ha señalado en ocasiones el Profesor Ramón Bayés ${ }^{(94)}$. Ahora bien, esta formación no asegura necesariamente que mejore la capacidad para detectar la angustia y otros trastornos psicológicos, ni la actitud para derivar adecuadamente al paciente al especialista.

De hecho, hay estudios pesimistas respecto a estos programas formativos: con un esquema semejante al utilizado por Lesley Fallowfield(80), Isabelle Merckaert y sus colaboradores ${ }^{(95)}$ estudiaron la capacidad de los médicos para detectar el malestar emocional, antes y después de un programa de formación; tras cinco meses del entrenamiento, no encontraron que dicha capacidad fuera diferente de la de otros médicos que no recibieron formación. Los autores señalan la necesidad de dirigir la formación a temas más específicos, en técnicas de apoyo emocional y a trabajar los factores que interfieren con la detección en el caso de los médicos.

Para terminar este apartado, y como contrapunto, hay que señalar que otros estudios como el de Mariette Mercier y sus colaboradores $^{(96)}$ encuentran buena concordancia entre las auto-evaluaciones que los pacientes realizan sobre su malestar/ bienestar psíquico, y la posterior evaluación que de ellos hacen los profesionales, si bien, en este caso las puntuaciones de los pacientes tendían a ser más extremas que las de los profesionales, tanto al indicar mayor bienestar o mayor malestar.

Por otra parte, no se puede considerar, de modo general, que el médico sea insensible al malestar psíquico del paciente. En ocasiones son la enorme presión asistencial con la que desarrollan su trabajo, y la falta de tiempo para atender adecuadamente a sus pacientes lo que produce ese desfase ${ }^{(61,97)}$, sin olvidar las veces en que son los pacientes, ya marcados con el estigma del diagnóstico, los que se muestran reacios a informar al médico sobre su malestar psíquico.

\section{Instrumentos de detección adecuados a la realidad asistencial}

No se trata aquí tanto del desarrollo de herramientas diagnósticas, con características psicométricas perfectas, sino de favorecer una evaluación y detección rápida y adecuada de los trastornos psicopatológicos, síntomas y otras necesidades psicológicas que pueden presentar los pacientes; adecuada, sobre todo, para salvar las dificultades o barreras que se han señalado, propias del paciente o del contexto asistencial, sin descuidar, por supuesto, que dichas herramientas tengan una suficiente validez.

En tal sentido, crece el interés por poner a disposición de los profesionales 
sanitarios herramientas de detección del malestar emocional y así derivar adecuada y rápidamente a los pacientes para tratamiento. Distintos autores hacen hincapié en esta actitud pragmática, que pretende los siguientes objetivos: 1) brindar a los pacientes orientación y ayuda psicológica desde las fases más tempranas del tratamiento médico ${ }^{(98-100)}$; 2) reducir los costes de los cuidados sanitarios ${ }^{(83,98,101)}$; y 3$)$ programar la atención sanitaria para los pacientes con elevado malestar psicológico, sin necesidad de largas entrevistas diagnósticas, o extensivos cuestionarios ${ }^{(69,98)}$.

Se destacan a continuación los principales instrumentos breves de detección de problemas psicológicos en pacientes con cáncer, y listados de síntomas.

La escala de ansiedad y depresión hospitalaria (HADS), desarrollada por Zigmond y Snaith ${ }^{(52)}$, es uno de los instrumentos de auto-informe más utilizados en los medios hospitalarios. Es sencilla y fácil de aplicar. Se caracteriza por eliminar los síntomas somáticos de la ansiedad y de la depresión que podrían deberse a la enfermedad física, incluyendo en su lugar otros índices más específicos de la vivencia psíquica del trastorno. Consta de una subescala de ansiedad y otra de depresión, con 7 ítems cada una, a los que el paciente responde en una escala Likert de 4 puntos (de 0 a 3), marcando la respuesta que mejor refleja su situación. Las puntuaciones para cada subescala van de 0-21, de 0-42 para la puntuación global o total. Según sus desarrolladores, el punto de corte 8-10 detecta los 'casos dudosos', y el de 11 o más, los 'casos clínicos', dando una medida de la severidad. El análisis factorial identifica dos factores, correspondientes a las subescalas de ansiedad y depresión. Su validez es de 0,70 y su fiabilidad alcanza un Alfa de Cronbach de 0,80 para cada una de las subescalas.

Según algunos autores ${ }^{(102)}$, su validez alcanza valores de sensibilidad/especifici- dad del $70 \% / 75 \%$ para la detección de posible trastorno de depresión mayor (tomando el punto de corte 19), y del 75\%/ $75 \%$ para la detección de posible trastorno adaptativo (tomando el punto de corte 13).

Según otros autores, no se puede considerar una herramienta de diagnóstico psiquiátrico sino de detección de síntomas de ansiedad y depresión ${ }^{(103)}$. Su sensibilidad, frente al DSM-III-R, alcanza el $80 \%$ en una muestra de pacientes orgánicos y del $100 \%$ en otra de pacientes psiquiátricos (tomando el punto de corte 8); en cambio, su valor predictivo positivo sólo alcanza al $17 \%$ para los primeros, y al $29 \%$ para los segundos; y sólo parece válida como indicador de la posibilidad de tener un trastorno depresivo.

El hecho de que las subescalas, por separado, no discriminen bien entre ansiedad y depresión, y que su valor predictivo sea escaso ${ }^{(53,104)}$, contribuye a que se prefiera usar la escala de forma global, tomando la puntuación total de la misma como medida de "malestar psíquico o emocional", proponiendo, además, puntos de corte variables (desde 10 hasta 20) según la enfermedad física, su severidad y la psicopatología de las poblaciones encuestadas. De esa manera muestra buena relación con escalas de calidad de vida ${ }^{(105)}$, y buena sensibilidad para detectar cambios durante el curso de la enfermedad o en respuesta a las intervenciones psicoterapéuticas ${ }^{(106)}$.

Además, esta herramienta nos interesa especialmente porque está validada en pacientes con cáncer de distinta de grave$\operatorname{dad}^{(14,102,107-109)}$, mostrando, en nuestro medio, una validez en torno al $70 \%$ usando los criterios DSM-IV como contraste ${ }^{(110)}$.

El listado de detección de problemas psicosociales (SIPP), desarrollado por Anna Braeken y sus colaboradores, en Holanda ${ }^{(111)}$, con el que hacen un seguimiento a los pacientes con cáncer durante el tratamiento de radioterapia, evaluando 
problemas físicos, psicológicos, sociales y sexuales. Consta de 24 ítems, y es utilizado también para facilitar la comunicación entre pacientes y radioterapeutas sobre malestar psicosocial, ayudar a un diagnostico precoz de los problemas psicosociales, y favorecer la derivación de pacientes a los psicólogos.

El listado breve de síntomas (BSI), desarrollado por Leonard Derogatis y Nick Melisaratos, primero como un listado de 53 ítems, y reducido posteriormente hasta $18^{(51,112-114)}$, eliminando algunas áreas no correlacionadas con la clínica habitual en pacientes con cáncer.

El test de calidad de vida en cancerología (FLIC), desarrollado por Mariette Mercier y colaboradores, en Francia ${ }^{(96)}$, validado por contraste de expertos. De 22 preguntas iniciales, encuentran que bastarían tres preguntas para predecir a más del $80 \%$ de los pacientes evaluados. Éstas eran: 1) ¿está usted satisfecho con su vida profesional o de cómo maneja sus trabajos?, 2) ¿cómo se siente usted físicamente? y 3) ¿en función de su enfermedad o de su tratamiento, está usted inquieto, preocupado?

El listado de malestar psíquico (PDI), desarrollado con rigor por Gabriella Morasso y sus colaboradores, en Italia ${ }^{(115)}$; consta de 13 ítems validados por contraste de expertos, y posteriormente en 3 submuestras: en una por contraste con el Inventario de Ansiedad Estado-Rasgo $(\mathrm{STAl})^{(116)}$; en otra por contraste con una entrevista clínica estructurada, con criterio CIE-10; y en una tercera, midiendo su capacidad discriminadora del malestar psíquico en pacientes con diferentes fases de la enfermedad, y como estimación de la prevalencia de trastornos psicopatológicos. Obtienen una sensibilidad del $75 \%$ y una especificidad del $85 \%$, a la hora de predecir posibles trastornos psicopatológicos en estos pacientes.

La escala de síntomas Edmonton (ESAS), desarrollada por Bruera y colabo- radores, en Canadá(117), de uso frecuente en la clínica médica para evaluar los siguientes 10 ítems: dolor, astenia, náuseas, depresión, ansiedad, somnolencia, apetito, bienestar, falta de aire, y dificultad para dormir, de los cuales se puntúa su presencia/ausencia, y su intensidad. También este tipo de escalas de síntomas emocionales y físicos siguen la tendencia actual a una mayor brevedad, para ajustarse a la realidad asistencial, como ocurre en la desarrollada por Zeeshan Butt y colaboradores, en USA ${ }^{(118)}$ en pacientes ambulatorios con cáncer, de sólo 4 ítems: 1 Fatiga, 2 Dolor, 3 Malestar emocional, y 4 Anorexia; y en otras más sencillas, como la escala visual analógica (EVA) desarrollada en nuestro medio por el grupo ASTHENOS ${ }^{(119)}$.

Estos instrumentos breves de evaluación destacados no están exentos de dificultades, debidas al tipo de formato, a las mediciones usadas, al tiempo requerido para administrarlos, y a la dificultad de los médicos para su interpretación. De ahí que se favorezca el desarrollo de herramientas aun más cortas, ultra-cortas, que tienen entre uno y 4 ítems, y requieren menos de dos minutos para completar$\mathrm{se}^{(82,120,121)}$ (tabla 1).

El termómetro de malestar psíquico (DT -Distress Thermometer), desarrollado por Andrew Roth y colaboradores, en USA y Francia ${ }^{(55)}$, uno de los instrumentos ultracortos actualmente utilizados. Se trata de una sola pregunta, presentada visualmente como un termómetro calibrado, que evalúa el malestar psíquico de los pacientes en una escala tipo Likert, de 0 a 10. Esta última puntuación indica el estado más alto y desfavorable, y se adopta el valor 5 como punto de corte, aunque su funcionamiento como detección no está revisado con entrevista diagnóstica.

Aunque el DT no tenga un punto de corte simple que maximice la sensibilidad y la especificidad, permite un efectivo y rápido reflejo de lo que sería el malestar 


\section{Tabla 1. Tipos de instrumentos de detección. Construido sobre Mitchell y colaboradores ${ }^{(120,82)}$}

\begin{tabular}{|l|l|l|l|}
\hline \multicolumn{1}{|c|}{ Tipo } & \multicolumn{1}{|c|}{ N ítems } & $\begin{array}{c}\text { Tiempo de res- } \\
\text { puesta }\end{array}$ & \multicolumn{1}{|c|}{ Ejemplos } \\
\hline Estándar & 15 o más & $>4$ minutos & $\begin{array}{l}\text { Inventario de depresión de Beck -BDI; } \\
\text { Inventario breve de síntomas -BSI18- y } \\
\text {-BSI53 }\end{array}$ \\
\hline Cortos & $5-14$ & $2-4$ minutos & $\begin{array}{l}\text { Escala de depresión postnatal de Edim- } \\
\text { burgo; Escala de ansiedad y depresión } \\
\text { hospitalaria -HADS }\end{array}$ \\
\hline Ultra-cortos & $1-4$ & $<2$ minutos & $\begin{array}{l}\text { Una única pregunta "esta Vd. deprimi- } \\
\text { do" } \\
\text { Termómetro de malestar psíquico -DT }\end{array}$ \\
\hline
\end{tabular}

psíquico en pacientes con cáncer, y permite usar puntos de corte distintos, para programar distintos niveles de intervención, estrategia que maximiza la utilidad del DT y de la entrevista asociada. Esta interpretación es solidaria con la idea del "Continuum" en la fenomenología de la clínica, y en particular en el contexto de las enfermedades físicas, como señalan Holland y Sheldon ${ }^{(71)}$.

EI DT fue desarrollado para valorar el malestar psíquico de un grupo de pacientes con carcinoma de próstata, y así derivarlos a tratamiento. Sus valores psicométricos fueron obtenidos por contraste con el HADS, con punto de corte 15. El $31 \%$ de los pacientes evaluados puntuaron por encima del punto de corte en cada una de las dos medidas y fueron enviados a psiquiatría de enlace para evaluación psiquiátrica; la cuarta parte de ellos tuvo diagnóstico psiquiátrico.

Estudios posteriores contrastando el DT y el HADS, encuentran buena correlación entre ambas herramientas ${ }^{(122)}$. El Grupo SE$\mathrm{POS}^{(92)}$ encuentra correlaciones de $r=0,50$ con la subescala de ansiedad, de $r=0,40$ con la subescala de depresión. Otros estudios $^{(123)}$ que utilizan el DT y el HADS, contrastándolos con un diagnóstico psiquiátrico -DSM-IV señalaban también la buena correlación entre ambas, pero el DT demuestra ser menos sensible y específico que el HAD para la detección de un diagnóstico psiquiátrico DSM-IV.

También se ha contrastado el DT con los ya referidos listados breves de síntomas BSI-53 y BSI-18 $8^{(112,114)}$, utilizados como medida de malestar emocional, con valores de Área Bajo Curva de 0,74, p<0,001 con el BSI-53, y de 0,80, $\mathrm{p}<0,01$ con el BSI$18^{(60)}$.

Este instrumento posee mayor potencial para la rutina clínica que las medidas más largas, pero tiene escasa precisión para detectar a los que tienen alto malestar psíquico. Según Alex Mitchell(120), mientras que los pacientes sin malestar son detectados en el $85 \%$ de los casos, la precisión del DT baja hasta el 55\% a la hora de detectar a los pacientes con malestar. Esto no quita para que, a juicio de otros autores como Paul Jacobsen ${ }^{(124)}$, pueda ser utilizado en la práctica para detectar a la mayoría de los casos con malestar, o con trastornos del estado del ánimo, y derivarlos a salud mental, a expensas de producir más errores falsos positivos que falsos negativos.

Como herramienta de detección de los pacientes con malestar emocional clínicamente significativo, el DT muestra una sensibilidad de moderada a buena $(0,65$ - 
$0,88)$ y una especificidad algo inferior $(0,59-0,85)^{(125)}$. En general, se ha señalado que los instrumentos ultra-cortos muestran baja sensibilidad y alta especificidad ${ }^{(126)}$.

El cuestionario de una sola pregunta para valorar la depresión "¿Está usted deprimido?" es otro de los instrumentos ultra-breves más utilizados, desde que Harvey Max Chochinov y sus colaboradores lo desarrollaran en 1997, para la valoración de pacientes hospitalizados, y en tratamiento paliativo ${ }^{(127)}$. Validado por contraste con el módulo de diagnóstico de depresión del Research Diagnostic Criteria $(\text { RDC })^{(128)}$, los autores encontraron una capacidad predictiva positiva en el $100 \%$ de los casos en que el paciente tenía al menos dos síntomas más de depresión clínica; y al contrario, los pacientes que respondían negativamente a este ítem, tampoco puntuaban en los otros criterios de depresión.

Dicha validez la obtenían por contraste con una entrevista semiestructurada para diagnóstico de depresión adaptada de la lista de trastornos afectivos y esquizofrenia (Schedule for Affective Disorders and Schizophrenia -SADS)(129), y el listado abreviado de depresión de -BDI-Short Form de Beck $^{(130)}$; pero estos resultados de validez deberían relativizarse teniendo en cuenta los márgenes de error del propio BDI, que en estudios previos mostraba un $21 \%$ de casos falsos negativos, y un $29 \%$ de falsos positivos $^{(131)}$.

El cuestionario para detectar trastornos adaptativos y depresión mayor, desarrollado por Nobuya Akizuki ${ }^{(123)}$, a semejanza del instrumento de Chochinov, utilizando en este caso la instrucción: "Por favor, gradúe su estado de humor durante la pasada semana, marcando en esta escala de 0 a 100". El valor 100 representa un humor relajado habitual, 60 es el punto de corte, y valores más bajos indican un estado de humor negativo. Extraída de un modelo de entrevista estructurada, esta pregunta es utiliza junto al DT, contrastando ambas con la escala HADS, que sirve de patrón oro, comprobando que la validez de dicho cuestionario para detectar trastornos adaptativos y depresión mayor es inferior a del HADS, pero comparable a la del DT.

El cuestionario de una sola pregunta para valorar la ansiedad, desarrollado posteriormente por Heather Davey y sus colaboradores ${ }^{(132)}$, es otro instrumento ultra-corto, orientado en este caso a detectar la ansiedad, con una sola pregunta: "Por favor, marque ¿Qué nivel de ansiedad tiene usted?", a la que los pacientes responden en una escala tipo Likert, de 5 valores, significando el 1 'nada de ansiedad' y el valor 5 'ansiedad extrema'. Los autores aplicaron dicho cuestionario a mujeres que acudían para diagnóstico o detección de cáncer de mama, junto a una escala analógica visual (VAS) de ansiedad, con valores de 0-100, y contrastando ambas con el inventario de Ansiedad Estado-Rasgo (STAI) ${ }^{(116)}$. Encuentran que dicha pregunta puede sustituir al cuestionario STAI, con respecto al cual hallaron una correlación Rho de Spearman de 0,75, siendo preferible como valoración rápida de la ansiedad cuando se tiene un espacio de tiempo limitado, o cuando no se quiere sobrecargar a los pacientes con una evaluación más larga.

Para finalizar este apartado, nos centraremos en instrumentos desarrollados en España, influidos también por esa tendencia a la brevedad para adecuarlos a situación asistencial y vital de estos pacientes.

Por la misma época en que Chochinov desarrollaba su conocida pregunta "¿está usted deprimido?", el profesor Bayés y sus colaboradores $^{(133)}$ demostraron que una simple pregunta sobre la percepción que tiene el paciente del paso del tiempo (largo, corto), seguida de otra sobre la razón de percibirlo así, era una forma sencilla de evaluar el bienestar o el sufrimiento percibido, a la vez que un modo de acercamiento terapéutico a la persona evaluada. 
Y más recientemente, Jorge Maté y colaboradores $^{(134)}$ han presentado un instrumento de Detección del Malestar Emocional (DME) en pacientes en la fase final de la vida. Parten del supuesto de que el estado de ánimo se ve continuamente influido por percepciones subjetivas que hacen variar la ecuación 'Realidad amenazante/Recursos existentes', y que dichas percepciones se pueden objetivar, con flexibilidad, disponiendo de una serie de preguntas: 1) sobre el estado de ánimo del paciente, éste debe elegir entre: "¿bien, mal, regular, o usted qué diría?"; 2) esa misma pregunta valorada en intensidad, con una escala que va desde el 0 ('muy mal'), al 10 ('muy bien'); 3) sobre la presencia de preocupaciones, y si responde afirmativamente, ¿de qué tipo? (económica, familiar, emocional, espiritual, somática, u otras); 4) "¿cómo lleva esa situación?", a la que se responde con valores de 0 a 10 (0 "no le cuesta nada", y 10 "le cuesta mucho"). Paralelamente el sanitario valora los signos externos del malestar emocional: "expresión facial, aislamiento, demanda constante de compañía/atención, alteraciones de comportamiento nocturno, y otros".

Este grupo de investigadores ${ }^{(135)}$ contrastaron posteriormente la validez del DME, con puntuación de corte $\geq 9$, con la escala HADS, con punto de corte mayor que 16, y con una escala de malestar emocional de 5 puntos, con el corte en igual o mayor de 3, encontrando valores de sensibilidad y especificidad superior al 75\%.

Y por último, para completar este listado de instrumentos de detección, señalar que en nuestra reciente tesis doctoral se presenta un instrumento ultra-corto, basando en dos preguntas de ansiedad y depresión, -PAD (Depósito Legal $B l-1209 / 2012)^{(1)}$, fundamentado en los instrumentos, ya señalados, de Chochinov ${ }^{(127)}$ y Davey ${ }^{(132)}$, que será próximamente publicado, junto a sus valores psicométricos de validez y predicción para distintos trastornos psicopatológicos establecidos con la Entrevista Neuropsiquiátrica Internacional (International Neuropsychiatric Interview -MINI) $(136,137)$, y criterios DSM-IV-TR ${ }^{(4)}$, en un grupo de 235 pacientes con tipos mixtos de cáncer, al inicio del tratamiento de radioterapia.

El paciente responde a las preguntas "¿Está usted ansioso?", y "¿Está usted deprimido?", de forma simple con un Sí, o un No, a lo que se añade, si se precisa, una escala Likert de intensidad, con distintos puntos de corte. Con ello se obtiene una puntuación para la medida de ansiedad (PAD-A) y otra para la depresión (PAD-D); así mismo, se puede utilizar la puntuación global de las dos preguntas (PAD-T) como una medida general del malestar emocional.

Establecida la validez del PAD, junto a la de las escalas HADS y DT, para estos mismos pacientes, por contraste con la entrevista MINI, algunos de los puntos de corte del PAD demuestran una adecuada validez, con Sensibilidad y Especificidad dentro del rango $75 \pm 5 \%$; siendo semejante al HADS, sobre todo para detectar los trastornos del estado del ánimo, y algo superiores al DT. En la tabla 2 siguiente se muestran distintos índices de validez y capacidad para pronosticar los trastornos del estado del ánimo (MINI).

Dentro de este mismo grupo diagnóstico, las Áreas Bajo Curva de la prueba ROC, son: para la subescala de ansiedad del HAD (HAD-A) 0,869 [p=0,000, IC $0,78-0,95]$; para la subescala de depresión (HAD-D) 0,870 [p=0,000, IC 0,792$0,949]$; para la escala total (HAD-T) 0,893 $[p=0,000$, IC 0,822-0,064]. Para el DT $0,770[p=0,000$, IC $0,686-0,854]$; para la pregunta de ansiedad (PAD-A) 0,823 $[p=0,000, I C$ 0,735-0,911]; para la pregunta de depresión (PAD-D) 0,840 [p=0,000, IC 0,739-0,942]; y para la puntuación conjunta de ambas preguntas (PAD-T) 0,863 $[p=0,000$, IC 0,776-0,950].

Este instrumento no resulta adecuado para la detección de los trastornos psico- 


\section{Tabla 2. Validez del HADS, DT y PAD, contrastadas con la entrevista MINI, para los Trastornos del estado de ánimo (Hernández, 2012)(1)}

\begin{tabular}{|l|l|l|l|l|l|l|l|l|l|l|l|}
\hline ESCALA & S & E & VPP & VPN & CPP & CPN & J & PSI & NND & NNP & OT \\
\hline HAD-A $>7$ &, 882 &, 749 &, 217 &, 988 & 3,51 &, 160 &, 60 &, 205 & 1,60 & 4,80 & 8 \\
\hline HAD-D $>7$ &, 766 &, 847 &, 267 &, 973 & 4,60 &, 350 &, 60 &, 240 & 1,60 & 4,10 & 9 \\
\hline HAD-T $>14$ &, 824 &, 800 &, 246 &, 983 & 4,12 &, 220 &, 60 &, 229 & 1,60 & 4,30 & 11 \\
\hline HAD-A $>10$ &, 706 &, 921 &, 414 &, 975 & 8,93 &, 320 &, 60 &, 389 & 1,60 & 2,50 & 12 \\
\hline HAD-D $>10$ &, 529 &, 953 &, 474 &, 962 & 11,38 &, 490 &, 50 &, 436 & 2 & 2,20 & 10 \\
\hline HAD-T >20 &, 766 &, 940 &, 480 &, 976 & 11,67 &, 310 &, 60 &, 456 & 1,60 & 2,10 & 13 \\
\hline DT >4 &, 706 &, 688 &, 152 &, 967 & 2,27 &, 430 &, 40 &, 119 & 2,50 & 8,40 & 1 \\
\hline PAD-A Sí/No &, 971 &, 400 &, 116 &, 994 & 1,62 &, 070 &, 40 &, 110 & 2,50 & 9 & 2 \\
\hline PAD-D Sí/No &, 941 &, 521 &, 134 &, 991 & 1,96 &, 110 &, 50 &, 125 & 2 & 8 & 4 \\
\hline PAD-T $>3$ &, 941 &, 540 &, 139 &, 991 & 2,04 &, 110 &, 50 &, 130 & 2 & 7,60 & 6 \\
\hline PAD-A $\geq 3$ &, 706 &, 777 &, 200 &, 971 & 3,16 &, 380 &, 50 &, 171 & 2 & 5,80 & 3 \\
\hline PAD-D $\geq 3$ &, 588 &, 902 &, 323 &, 965 & 6,02 &, 460 &, 50 &, 288 & 2 & 4,30 & 7 \\
\hline PAD-T $>4$ &, 706 &, 795 &, 214 &, 972 & 3,45 &, 370 &, 50 &, 186 & 2 & 5,30 & 5 \\
\hline
\end{tabular}

Leyenda: $S=$ Sensibilidad; $E=$ Especificidad; VPP.=Valor predictivo positivo; $V P N .=$ Valor predictivo negativo; $C P P .=$ Cociente de probabilidad positivo (razón de verosimilitud); $C P N .=$ Cociente de probabilidad negativo; $J=$ Índice J de Youden; PSI. = Predictive Summary Index; NND = Number Needed to Diagnosig; NNP = Number Needed to Predict. OT = Orden jerárquico Total (a partir de la puntuación del orden jerárquico de cada índice).

patológicos, pero sí para detectar el malestar emocional; logrando además, por su brevedad, responder a las necesidades que se vienen reclamando para instrumentos semejantes: que sea eficaz para evaluar aspectos relevantes para el paciente; que facilite la derivación a un profesional para una evaluación posterior y que posibilite efectos terapéuticos; que evite los efectos iatrogénicos; que sea breve, sencillo y fácil de administrar por profesionales sanitarios de diferente formación; que explore la situación en un periodo previo breve; que se pueda repetir su uso para reevaluar el malestar según avanza la enfermedad.

Las críticas al uso de instrumentos tan simples y breves para la detección del malestar psíquico y de la salud mental de estos pacientes, llegan en primer lugar de los clínicos que defienden la necesidad de la evaluación psicopatológica mediante entre- vistas estructuradas, con criterios DSM-IV, más precisos y eficaces. Así, por ejemplo, James Coyne y colaboradores ${ }^{(138)}$, en su estudio con mujeres con alto riesgo de tener un cáncer hereditario de mama y de ovario, mostraban que la conocida escala de síntomas de ansiedad y depresión Hopkins Symptom Checklist (HSCL-25)(139), resultaba claramente ineficaz frente a una valoración con la entrevista estructurada del DSM-IV: menos del $10 \%$ de las mujeres detectadas con malestar psíquico según la escala reunía criterio DSM-IV de trastorno psíquico; concluyendo que estas evaluaciones pueden generar un mal uso de los recursos.

El siguiente tipo de críticas se refiere a la incapacidad de estos instrumentos para mantener su validez en todo tipo de pacientes; por ejemplo, a la hora de diagnosticar, detectar o predecir trastornos adaptativos en pacientes en trasplante de médula ósea ${ }^{(140)}$. 
Un tercer tipo de críticas se refiere a la dificultad para extrapolar el uso de estos cuestionarios a culturas distintas de aquellas en las que se desarrollaron. Así ocurre con el instrumento ultra-breve ya presentado de Chochinov ${ }^{(127)}$ para valorar la depresión, que mostraba una sensibilidad y especificidad de $100 \%$ en la población de pacientes terminales norteamericanos: al estudiar este mismo instrumento en una muestra de pacientes japoneses en tratamiento de radioterapia, no se encuentran, ni de lejos, dichos valores de sensibilidad y especificad ${ }^{(141)}$, ni tampoco en estudios con enfermos terminales, o en tratamiento paliativo japoneses ${ }^{(142)}$, o europeos ${ }^{(143,144)}$. Lo cual podría ser una señal no sólo de la diferente validez del test en función de la cultura, sino también de deficiencias internas del instrumento.

Como resumen, podríamos decir, que estas herramientas presentan dos desventajas: su validez mas bien modesta, y que desconocemos la validez de muchas de ellas para todo tipo de pacientes con cáncer, o en distintos momentos de la evolución de la enfermedad.

Pero cabe recordar que detección y evaluación clínica son cosas distintas, y no tienen por qué ir reñidas. La detección identifica de un modo rápido qué pacientes pueden tener dificultades importantes en su proceso de enfrentar la enfermedad y de adaptarse a los tratamientos. La evaluación clínica efectúa una serie de tareas, a partir de un vínculo mayor con el paciente, que incluyen la comprensión dinámica del paciente, el establecimiento de un diagnóstico incluyendo la estimación de la gravedad, y el diseño de una acción de cambio, o el inicio de la relación terapéutica. En el contexto clínico, los métodos de detección son más breves, y pragmáticos, pues permiten incorporar tempranamente las intervenciones psicológicas o sociales en el tratamiento integral del paciente con cáncer, y además pueden repetirse con facilidad, para dar cuenta del estado cambiante del malestar emocional en estos pacientes.

Además, conviene distinguir que las evaluaciones clínicas basadas en las entrevistas estructuradas, tienden a definir el malestar psíquico a partir de criterios psicopatológicos, mientras que los instrumentos breves de detección no buscan, inicialmente al menos, identificar trastornos psicopatológicos, sino conocer y ofrecer intervenciones para el amplio espectro de circunstancias que Ilevan al malestar psíquico de los pacientes con cáncer. Este aspecto práctico es, quizás, la razón del amplio uso actual del DT, como destaca la revisión de Andrea Vodermaier ${ }^{(145)}$, aunque, como se ha señalado, tanto su fiabilidad como validez sea sólo moderada (tabla 3) $)^{(146)}$.

\section{REFLEXIONES A MODO DE CONCLUSIÓN}

Los estudios revisados muestran la alta frecuencia de problemas de salud mental y de síntomas de ansiedad, depresión y malestar emocional en pacientes con cáncer, en mayor medida que en la población general. Pero las diferencias metodológicas entre los distintos estudios, respecto a las muestras evaluadas, y la falta de homogenización de los conceptos e instrumentos utilizados, impide concluir sobre la prevalencia real de estos problemas.

Ello contribuye a que actualmente la asistencia psicológica específica que se presta a estos pacientes sea muy deficitaria, por lo que muchos pacientes que podrían necesitar una ayuda o un tratamiento psicológico específico no lo reciben, probablemente ni siquiera son detectados. Las razones pueden estar en una escasa implementación de los recursos psicológicos en los hospitales, y, a la inversa, los escasos recursos puede sustentarse en un deficitario sistema de detección y evaluación, que ponga en evidencia la necesidad de aquéllos. 


\section{Tabla 3. Criterios de validez de instrumentos cortos para la detección del malestar emocional, en el contexto del cáncer. Tomada de Mitchell(146).}

\begin{tabular}{|c|c|c|c|c|}
\hline $\begin{array}{l}\text { Validez en muestras múltiples, } \\
\text { independientes. }\end{array}$ & $\begin{array}{l}\text { Sensibilidad } \\
(95 \%, \text { I.C. })\end{array}$ & $\begin{array}{l}\text { Especificidad } \\
(95 \%, \text { I.C. })\end{array}$ & $\begin{array}{l}\text { Capacidad } \\
\text { para } \\
\text { encontrar } \\
\text { casos } \\
(\text { AUC+) }\end{array}$ & $\begin{array}{l}\text { Capacidad } \\
\text { para la } \\
\text { detección } \\
\text { (AUC-) }\end{array}$ \\
\hline $\operatorname{HADS}(\mathrm{N}=13)$ & $\begin{array}{l}70,4 \% \\
(56,1 \%-82,9 \%)\end{array}$ & $\begin{array}{l}80,6 \% \\
(72,8 \%-87,4 \%)\end{array}$ & 0,704 & 0,662 \\
\hline $\mathrm{DT}(\mathrm{N}=4)$ & $\begin{array}{l}78,5 \% \\
(69,8 \%-86,1 \%)\end{array}$ & $\begin{array}{l}67,4 \% \\
(60,1 \%-74,3 \%)\end{array}$ & 0,643 & 0,682 \\
\hline $\begin{array}{l}\text { Una simple pregunta verbal (Single } \\
\text { Verbal Question) }(\mathrm{N}=4)\end{array}$ & $\begin{array}{l}67,3 \% \\
(51,0 \%-81,6 \%)\end{array}$ & $\begin{array}{l}78,9 \% \\
(58,3 \%-93,7 \%)\end{array}$ & 0,687 & 0,648 \\
\hline \multicolumn{5}{|l|}{ Validez en muestras únicas. } \\
\hline $\begin{array}{l}\text { Listado de malestar psicológico } \\
\text { (Psychological Distress Inventory) }(\mathrm{N}=1)\end{array}$ & $\begin{array}{l}68,0 \% \\
(54,2 \%-79,2 \%)\end{array}$ & $\begin{array}{l}90,2 \% \\
(81,9 \%-94,9 \%)\end{array}$ & 0,787 & 0,667 \\
\hline DT e IT (Impact Thermometer) $(\mathrm{N}=1)$ & $\begin{array}{l}81,3 \% \\
(74,6 \%-90,3 \%)\end{array}$ & $\begin{array}{l}82,1 \% \\
(75,3 \%-87,3 \%)\end{array}$ & 0,734 & 0,730 \\
\hline $\begin{array}{l}\text { 2-Preguntas: "¿Tienes usted falta de } \\
\text { interés o capacidad de disfrute?" o } \\
\text { ¿Está usted deprimido?" (2-Question: } \\
\text { "Have you lost interest or pleasure?" or } \\
\text { "Are you depressed?") }(\mathrm{N}=1)\end{array}$ & $\begin{array}{l}68,1 \% \\
(53,8 \%-79,6 \%)\end{array}$ & $\begin{array}{l}93,5 \% \\
(84,6 \%-97,5 \%)\end{array}$ & 0,831 & 0,673 \\
\hline
\end{tabular}

Abreviaturas: $A \cup C+=$ Área bajo curva positiva; $A \cup C$-, Área bajo curva negativa.

Las dificultades o barreras para una adecuada detección de estos problemas, se deben a factores que afectan al propio paciente, a los profesionales de la salud, y al contexto asistencial actuales.

Las investigaciones más interesantes, orientadas desde la clínica, para solucionar algunas de estas barreras, se centran en dos vías: una es la formación a los profesionales de la salud en habilidades de comunicación y reconocimiento de problemas psicológicos, con resultados contradictorios y de reducida eficacia, quizás por ser una formación escasa, basada en breves cursos, que no suponen una capacitación suficiente, y dejan sin resolver algunas de las barreras señaladas del lado de los profesionales. En la experiencia cotidiana, vemos en un extremo al profesional que se sigue resistiendo a hablar con sus pacientes de los problemas psicológicos o emocionales (aun teniendo noticia de ellos), y en el otro extremo el riesgo de que el profesional identifique la parte con el todo, y reduzca 'lo psicológico' al curso o a la herramienta que se le facilita, y a partir de ahí, como dice el refrán, con un martillo en la mano sólo ve clavos, sin considerar que hay herramientas adecuadas a cada cosa, y que existe el especialista psicólogo para derivar al paciente.

La otra vía es la implementación de herramientas de cribado o detección adecuadas a la realidad asistencial y vital de los pacientes, fácilmente aplicables y repetibles con facilidad; que ha mostrado resultados mejorables, sobre todo en los aspectos psicométricos de validez y precisión diagnóstica, que es más bien modesta; aunque permiten elegir distin- 
tos puntos de corte, que faciliten bien la sensibilidad, o bien la especificidad.

El concepto de malestar emocional, es impreciso, pero sirve para reconocer una realidad clínica, muy prevalente en los pacientes oncológicos, igualmente poco atendida, y que supone, en cuanto a factor de riesgo, un elemento diferenciador de la evolución psicopatológica de estos pacientes. Quizás el mayor interés de este concepto radica en el intento de homologar, en las distintas instituciones asistenciales, un modo de detección rápida, que facilite la orientación a los distintos niveles de intervención, incluida la atención en salud mental.

A pesar de esas ventajas prácticas, la vía de las herramientas presentan dificultades: la mayor es que se detecten problemas que no se puedan atender, y no exista la posibilidad de enviarlo al psicólogo, una de la razones que frustran a los profesionales que podrían utilizarlas (Mitchell, 2008); con todo, parece una vía más útil que la de la formación al sanitario. Faltan estudios que integren ambas vías, y es preciso seguir evaluando su validez y precisión en la detección de la salud mental, el malestar emocional, y las necesidades psicológicas reales de los pacientes, y su utilidad clínica, es decir, la mejora que supone en la atención.

Todo ello llevaría a que los pacientes con mayores niveles de malestar y psicopatología fueran atendidos en las consultas, y acudieran al psicólogo todos aquellos que lo necesitaran. A este último le correspondería la evaluación clínica y extensa, y el diseño y desarrollo del programa asistencial adecuado a cada caso.

En la línea del compromiso actual de investigación-en-acción, urge investigar la eficacia de estos instrumentos, en cuanto a optimización o mejora de la atención, si contribuye a resolver el problema de cómo proporcionar una atención psicológica inmediata, precoz, y eficiente (en términos coste/beneficios), efectiva para aplicarse en el contexto, y que se traduzca en actuaciones eficaces.

\section{APORTACIONES PARA LA PRÁCTICA}

De este trabajo de revisión, surgen dos propuestas complementarias:

Habría que incluir en todos los hospitales un instrumento, como los descritos, de detección del malestar y derivación al especialista psicólogo, ya que el riego de malestar emocional en el paciente de cáncer es alto, y eso no debería ser ignorado. Además, los instrumentos son breves y económicos, y de fácil aplicación.

Habría que promover una mayor coordinación y adaptabilidad del psicólogo con los equipos médicos, para favorecer la detección temprana de los problemas de salud mental y malestar emocional y prestar la asistencia específica y precoz a los pacientes que lo precisen. Esa coordinación permitiría un trabajo interdisciplinar, de cara al paciente, y al propio equipo.

AGRADECIMIENTOS: Este trabajo forma parte de la investigación realizada en el Hospital Universitario de Basurto, sobre "Evaluación psicológica de pacientes con cáncer en tratamiento de radioterapia", presentada este año como tesis doctoral en la Universidad Complutense de Madrid. Agradecemos a los profesionales de los Servicios de Psiquiatría y Psicología clínica y de Oncología Radioterápica su colaboración, así como a las psicooncólogas becarias que ayudaron en su desarrollo. Así mismo agradecemos la subvención recibida de la Unidad de Investigación de dicho Hospital y la Fundación Vasca de Innovación e Investigación Sanitarias - BIOEF (BIOEF07/037/C y BIOEF10/006), y al Colegio Oficial de Psicólogos de Madrid, el haberla honrado con el primer premio de Psicología Rafael Burgaleta. 


\section{REFERENCIAS BILIOGRÁFICAS}

1. Hernández M. Evaluación psicológica de los pacientes oncológicos en tratamiento de radioterapia. [Tesis doctoral]. Madrid: Universidad Complutense de Madrid; 2012.

2. Derogatis LR, Morrow GR, Fetting J, Penman D, Piasetsky S, Schmale AM. et al. The prevalence of psychiatric disorders among cancer patiens. JAMA 1983;249:751-7. Doi: 10.4103/0973-1482.27590.

3. Hernández M, Cruzado JA, Arana Z. Problemas psicológicos en pacientes con cáncer: dificultades de detección y derivación al psicooncólogo. Psicoooncología 2007;4(1):179-91.

4. American Psychiatric Association. Diagnosis Classification DSM-IV-TR. Versión española López-Ibor JJ y Valdés $M$, directors. Manual diagnóstico y estadístico de los trastornos mentales ( $4^{\mathrm{a}}$ ed. Texto revisado) Barcelona: Masson; 2002.

5. Gil FL, Costa G, Pérez FJ, Salamero M, Sánchez N, Sirgo A. Adaptación psicológica y prevalencia de trastornos mentales en pacientes con cáncer. Med Clin (Barc) 2007;(0):1-3.

6. Van't Spijker A, Trijsburg RW, Duivenvoorden HJ. Psychological Sequelae of Cancer Diagnosis: A meta-analytical review of 58 Studies after 1980. Psychosom Med 1997;59(3):280-93.

7. Massi MJ, Lloyd-Williams M, Irving G, Miller K. The prevalence of depression in people with cancer. In: Kissane D, Maj M, Sartorius $\mathrm{N}$, editors. Depression and Cancer. Chichester: Wiley-Blackwell; 2010. p. 1-36.

8. Martínez M, Dolz M, Alonso J, Luque I, Palacín C, Bernal M. et al. Prevalencia de los trastornos mentales y factores asociados: resultados del estudio ESEMedEspaña: European Study of the Epidemiology of Mental Disorders-España. Med Clin 2006;12(126):445-51.

9. Pessin H, Amakawa L, Breitbart WS. Suicide. En Holland JC, Breitbart W, Jacobsen P, edi- tores. Psycho-Oncology. New York - Oxford: Oxford University Press; 2010. p 319-23.

10. Breitbart W. Suicide in cancer patients. Oncology 1987;1:49-53.

11. Breitbart W, Krivo S. Suicide. In: Holland JC, editor. Psycho-Oncology. New York Oxford: Oxford University Press; 1998. p. 541-7.

12. Busch KA, Clark DC, Fawcett J, Kravitz $\mathrm{H}$. Clinical features of inpatient suicide. Psych Annals 1993;23(5):256-62.

13. Gabilondo A, Alonso J, Pinto-Meza A, Vilaguta G, Fernández A, Serrano-Blanco A, et al. Prevalencia y factores de riesgo de las ideas, planes e intentos de suicidio en la población general española. Resultados del estudio ESEMeD. Med Clin (Barc) 2007;129(13):494-500.

14. Ibbotson $T$, Maguire $P$, Selby $T$, Priestman $T$ y Wallace L. Screening for anxiety and depresion in cancer patients: the effects of disease and treatment. Eur J Cancer 1994;30A:37-40.

15. Noyes RJr, Holt CS, Massie MJ. Anxiety Disorders. En Holland JC, editor. PsychoOncology. New York - Oxford: Oxford University Press; 1998. p. 548-63.

16. Levin TT, Alici Y. Anxiety disorders. En Holland JC, Breitbart W, Jacobsen P, editores. Psycho-Oncology. New York - Oxford: Oxford University Press; 2010. p. 324-31.

17. Snyder S, Strain JJ. Diagnostic instability in psychiatric consultations. Hosp Community Psychiatr 1990;41:10-3.

18. Akechi T, Okuyama T, Sugawara $\mathrm{Y}, \mathrm{Na}-$ kano T. Major depression, adjustment disorders, and post-traumatic stress disorder in terminally ill cancer patients: Associated and predictive factors. J Clin Oncol 2004;22(10):1957-65.

19. Strain JJ. Adjustment disorders. In: Holland JC, editor. Psycho-Oncology. New York Oxford: Oxford University Press; 1998. p. 509-17

20. DeFlorio M, Massie MJ. Review of depression in cancer: Gender differences. Depression 1995;5:343-59. 
21. Massie MJ, Lloyd-Williams M, Irving G. Miller K. The prevalence of depression in people with cancer. In: Kissane D, Maj M, Sartorius N, editores. Depression and cancer. Chichester: Wiley-Blackwell; 2010.p.1-36.

22. Miller K, Massie M. Despressive Disorders. En Holland JC, Breitbart W, Jacobsen P, editores. Psycho-Oncology. New York Oxford: Oxford University Press; 2010. p. 311-8.

23. Bukberg J, Penman D, Holland JC. Depression in hospitalized cancer patients. Psychosom Med 1984;46:199-212.

24. Craig T, Abeloff MD. Psychiatric symptomatology among hospitalized cancer patients. Am J Psychiat 1977;131:1323-7.

25. Plumb M, Holland JC. Comparative studies of psychological function in patients with advanced cancer. 1. Self-reported depressive symptoms. Psychosom Med 1977;39:264-76.

26. Grassi L, Indelli M, Mazola M, Maestri A, Santini A, Piva E, et al. Depressive symptoms and quality of life in home-careassisted cancer patients. J Pain Sympton Manage 1996;12(5):300-7.

27. Massie MJ. Prevalence of depression in patients with cancer. J Natl Cancer Inst Monogr. 2004;32:57-71.

28. Joffe RT, Rubinow DR, Denicoff KD, Maher $M$, Sindelar WF. Depression and carcinoma of the pancreas. Gen Hosp Psychiatry 1986;8:241-5.

29. Massie MJ, Popkin MK. Depressive disorders. In Holland JC, Massiey MJ, editors. Psycho-Oncology. New York - Oxford: Oxford University Press. 1998. p. 518-40.

30. Akechi T, Okamura H, Kugaya A, Nakano T, Nakanishi T, Akizuki N, et al. Suicidal ideation in cancer patients with major depression. Jpn J Clin Oncol 2000;30(5):2214.

31. Bjorkenstam C, Edberg A, Ayoubi S, Rosen M. Are cancer patients at higher suicide risk than the general population?. Scand J Public Health 2005;33(3):208-14.
32. Bill-Axelson A, Garmo H, Lambe M, Bratt O, Adolfsson J, Nyberg U, et al. Suicide risk in men with prostate-specific antigendetected early prostate cancer: A nationwide population-based cohort study from PCBaSe Sweden. Eur Urol 2009;57:390-5. Doi:10.1016/j.eururo.2009.10.035.

33. Hinton J. Psychiatric consultation in fatal illness. Proc R Soc Med 1972;65(11):10351038.

34. Spiegel D, Glafkides MC. Effects of group confrontation with death and dying. Int J Group Psychother 1983;33(4):433-47.

35. Fallowfield LJ, Hall A. Psychosocial and sexual impact of diagnosis and treatment of breast cancer. $\mathrm{Br}$ Med Bull 1991;47(2):388-99.

36. Tross S, Holland J. Psychological sequelae in cancer survivors. In Holland JC, Rowland $\mathrm{JH}$, editors. Psycho-Oncology: Psychological care of the patient with cancer. New York, NY, US: Oxford University Press; 1989. p. 101-6.

37. Cassileth BR, Lusk EJ, Strouse TB, Miller DS, Brown LL, Cross PA, et al. Psychosocial status in chronic illness: A comparative analysis of six diagnostic groups. $\mathrm{N}$ Engl J Med 1984;311:506-11.

38. Cassileth BR, Lusk EJ, Walsh WP. Anxiety levels in patients with malignant disease. Hospice J 1986;2:57-69.

39. Noyes R, Kathol RG, Debelius-Enemark P, Williams J, Mutgi A, Suelzer MT, et al. Distress associated with cancer as measured by the Illness Distress Scale. Psychosomatics 1990;31:321-30.

40. Mahon SM, Cella DF, Donovan MI. Psychosocial adjustment to recurrent cancer. Oncolo Nurs Forum 1990;17(3):47-54.

41. Ahles TA, Blanchard EB, Rusckdeschel JC. The multidimensional nature of cancerrelated pain. Pain 1983;17:277-88.

42. Grassi L, Travado L, Gil F, Sabato S, Rossi E, grupo SEPOS. Psychosocial morbidity and its correlates in cancer patients of the Mediterranean area: Findings from the Southern European Psycho-Oncology 
Study. J Affect Disord 2004;83:243-8. Doi:10.1016/j.jad.2004.07.004.

43. Carroll BT, Kathol RG, Noyes RJr, Wald TG, Clamon GH. Screening for depression and anxiety in cancer patients using the Hospital Anxiety and Depression Scale. Gen Hosp Psychiatry 1993;15(2):69-74.

44. Ford S, Lewis S, Fallowfield L. Psychological morbidity in newly referred patients with cancer. J Psychosom Res 1995;39(2):193-202.

45. Rodríguez Vega B, Ortiz A, Barrero A, Avedillo C, Sánchez-Cabezudo A, Chinchilla C. Síntomas de ansiedad y depresión en un grupo de pacientes oncológi$\cos$ y en sus cuidadores. Eur J Psychiatry 2002;16(1):27-38. Doi:10.4321/S1579699X2002000100004.

46. Bultz BD, Carlson LE. Emotional distress: the sixth vital sign in cancer care. J Clin Oncol 2005;23(26):6440-1.

47. National Comprehensive Cancer Network (NCCN). Practice guidelines for the management of psychosocial distress. Oncology 1999;13:113-47.

48. Holland J. Preliminary guidelines for the treatment of distress. Oncology 1997;11:109-14.

49. National Comprehensive Cancer Network (NCCN). Angustia. Guías de tratamiento para pacientes. [Internet] 2004 [Versión I/ Julio 2004, citado 3 de mayo de 2005]. Disponible en: http://www.nccn.org/patients/patient_gls/_spanish/pdf/NCCN_angustia.pdf

50. Kessler RC, Chiu WT, Demler O, Walters EE. Prevalence, Severity, and Comorbidity of 12-Month DSM-IV Disorders in the National Comorbidity Survey Replication. Arch Gen Psychiatry 2005;62(6):617-27. Doi:10.1001/archpsyc.62.6.617.

51. Derogatis LR, Melisaratos N. The Brief Symptom Inventory (BSI): An introductory report. Psychol Med 1983;13:595-606. Doi:10.1017/S0033291700048017.

52. Zigmond A, Snaith, R. The Hospital Anxiety and Depression Scale.
Acta Psychiatr Scan. 1983;67:36170. Doi:10.1111/j.1600-0447.1983. tb09716.x.

53. Spinhoven PH, Ormel J, Sloekers PP, Kempen GI, Speckens AE, Van Hemert AM. A validation study of the Hospital Anxiety and Depression Scale (HADS) in different groups of Dutch subjects. Psychol Med 1997;27:363-370. Doi:10.1017/ S0033291796004382.

54. Kobayashi M, Ohno T, Noguchi W, Matsuda A, Matsushima E, Kato S, et al. Psychological distress and quality of life in cervical cancer survivors after radiotherapy. do treatment modalities, disease stage, and self-esteem influence outcomes? Int J Gynecol Cancer 2009;19(7):1264-8.

55. Roth AJ, Kornblith AB, Batel-Copel L, Peabody E, Scher HI, Holland J. Rapid screening for psychologic distress in men with prostate carcinoma: A pilot study. Cancer 1998;82(10):1904-8.

56. Henselmans I, Helgeson VS, Seltman H, Vries J, Sanderman R, Ranchor AV. Identification and prediction of distress trajectories in the first year after a breast cancer diagnosis. Health Psychol 2010;29(2):1608. Doi:10.1037/a0017806.

57. Zabora J, BrintzenhofeSzoc K, Curbow B, Hooker C, Piantadosi S. The prevalence of psychological distress by cancer site. Psychooncology 2001;10(1):19-28. Doi: 10.1002/1099-1611(200101/02)10:1< 19::AID-PON501>3.0.CO;2-6.

58. Hegel MT, Moore CP, Collins ED, Kearing $\mathrm{S}$, Gillock KL, Riggs RL, et al. Distress, psychiatric syndromes, and impairment of function in women with newly diagnosed breast cancer. Cancer 2006;107(12):292431. DOI: $10.1002 /$ cncr.22335.

59. Schain WS, D'Angelo TM, Dunn ME, Lichter AS, Pierce LJ. Mastectomy versus conservative surgery and radiation therapy: psychosocial consequences. Cancer 1994;73(4):1221-8.

60. Hoffman KE, McCarthy EP, Recklitis CJ, $\mathrm{Ng}$ AK. Psychological distress in long-term 
survivors of adult-onset cancer. Results from a nacional survey. Arch Intern Med 2009;169(14):1274-81. Doi:10.1001/archinternmed.2009.179.

61. Holland J. Update: NCCN practice guidelines for the treatment of distress. Oncology 1999;13(11A):459-507.

62. Spiegel D, Classen C. Group therapy for cancer patients. A research-based handbook of psychosocial care. New York: Basic Books; 2000.

63. Faller $H$, Bulzebruck $H$, Drings $P$, Lang $\mathrm{H}$. Coping, distress, and survival among patients with lung cancer. Arch Gen Psychiatry 1999;56(8):756-62.

64. Goodwin PJ, Leszcz M, Ennis M, Koopmans J, Vincent L, Guther H, et al. The effect of group psychosocial support on survival in metastatic breast cancer. N Engl J Med 2001;345(24):1719-26.

65. Prieto JM, Atala J, Blanch J, Carreras E, Rovina $M$, Cirena $E$, et al. Role of depression as a predictor of mortality among cancer patients after stem-cell transplantation. J Clin Oncol 2005;23(25):6063-71.

66. Spiegel D. Cancer and depression. $\mathrm{Br}$ J Psychiatry 1996;168(Suppl 30):109-16.

67. Spiegel D, Bloom JR, Kraemer HC, Gottheil E. Effect of psychosocial treatment on survival of patients with metastatic breast cancer. Lancet 1989;2(8668):888-91.

68. Back AL, Arnold RM, Baile WL, Tulsky JA, Fryer-Edwards K. Approaching difficult communication tasks in oncology. CA: Cancer J Clin 2005;55(3):164-77.

69. Zabora JR. Screening Procedures for Psychosocial Distress. En Holland JC, editor. Psycho-Oncology. New York - Oxford: Oxford University Press; 1998. p. 653-61.

70. Cruzado JA. Tratamiento psicológico en pacientes con cáncer. Madrid: Síntesis; 2010.

71. Holland J, Sheldon L. La cara humana del cáncer. Vivir con esperanza, afrontar la incertidumbre. Barcelona: Herder; 2003.

72. Baker-Glenn EA, Park B, Granger L, Symonds P, Mitchell AJ. Desire for psy- chological support in cancer patients with depression or distress: validation of a simple help question. Psychooncology. 2011;20:525-531 Doi: 10.1002/ pon.1759.

73. Kreitler S, Chaitchik S, Kreitlers H. Repressiveness: Cause or result of cancer? Psychooncology 1993;2:43-54.

74. Watson M, Greer S, Blake S, Shrapnell K. Reaction to a diagnosis of breast cancer: relationship between denial, delay and rates of psychological morbidity. Cancer 1984;53:2008-12.

75. Shedler J, Mayman M, Manis M. The illusion of mental health. Am Psychol 1993; 48:1117-31.

76. Schavelzon J, Bleger L, Bleger IL, Luchina I, Langer, M. Cáncer: enfoque psicológico. Buenos Aires: Galerna; 1978.

77. Holland JC, Rowland JH, editores. Handbook of psychooncology: Psychological care of the patient with cancer. New York, NY, US: Oxford University Press; 1989.

78. Holland, JC. History of Psycho-Oncology: Overcoming attitudinal and conceptual Barriers. Psychosom Med 2002;64:20621.

79. Kagan AR, Clifton R. Psychologically highrisk patients in a radiation oncology department. Am J Clin Oncol 2002;25(4):425-7.

80. Fallowfield L, Ratcliffe D, Jenkins V, Saul J. Psychiatric morbidity and its recognition by doctors in patients with cancer. $\mathrm{Br}$ J Cancer 2001;84:1011-5.

81. Goldberg D, Williams P. A User's Guide to the General Health Questionnaire. Windsor: NFER-Nelson; 1988.

82. Mitchell AJ, Kaar S, Coggan C, Herdman J. Acceptability of common screening methods used to detect distress and related mood disorders: preferences of cancer specialists and non-specialists. Psychooncology 2008;17:226-36. Doi: 10.1002/pon.1228.

83. Payne DK, Hoffman RG, Theodoulou M, Dosik M, Massie MJ. Screening for anxiety and depression in women with breast 
cancer. Psychiatry and medical oncology gear up for managed care. Psychosomatics 1999;40(1):64-9.

84. Ronson A, Chapeaux N, Body JJ. Le trouble de I'adaptation avec humeur depressive chez les patients cancereux: Comment le diagnostiquer et quand le traiter? [Adjustment disorders with depressed mood in patients with cancer: How to diagnose and when to treat?]. Rev Fr Psychooncologie 2003;2(1-2):25-9.

85. McDaniel JS, Nemeroff CB. Depression in the cancer patient: Diagnostic, biological, and treatment aspects. In: Chapman CR, Foley KM, editors. Current and emerging issues in cancer pain: research and practice. New York: Raven Press; 1993.p.119.

86. Álvarez MA. Evaluación e intervención del riesgo suicida. Servicio de Asistencia Psiquiátrica de la Comunidad de Castilla y León. En: I Jornadas en seguridad del paciente en salud mental. Conferencia; febrero de 2011; Cáceres; 2011.

87. Passik SD, Lowery AE. Recognition and screening of depression in people with cancer. In: Kissane D, Maj M, Sartorius N, editors. Depression and Cancer. Chichester: Wiley-Blackwell; 2010.p.81-100.

88. Cohen-Cole SA, Brown FW, McDaniels JS. Assessment of depresion and grief reactions in the medically ill. In: Stoudemire A, Fogel BS, editors. Psychiatric care of the medical patient. New York: Oxford University Press; 1993. p. 53-69.

89. Passik SD, Dugan W, McDonald MV, Rosenfeld B, Theabald DE, Edgerton S. Oncologists' recognition of depression in their patients with cancer. J Clin Oncol 1998;16(4):1594-600.

90. Passik SD, Kirsh KL, Theobald D, Donaghy K, Holtsclaw E, Edgerton S, et al. Use of a depression screening tool and a fluoxetine-based algorithm to improve the recognition and treatment of depression in cancer patients: a demonstration project. J Pain Symptom Manage 2002;24:318-27.
91. McLachlan SA, Allenby A, Matthews J, Wirth A, Kissane DW, Bishop M, et al. Randomized trial of coordinated psychosocial interventions based on patient self-assessments versus standard care to improve the psychosocial functioning of patients with cancer. J Clin Oncol 2001;19:4117-25.

92. Grassi L, Travado L, Gil F, Campos R, Lluch P, Baile W. A communication intervention for training Southern European Oncologists to recognize psychosocial morbidity in cancer. I- Development of the model and preliminary results on physicians'satisfaction. J Cancer Educ 2005;20:79-84.

93. Travado L, Grassi L, Gil F, Ventura C, Martins C, grupo SEPOS. La comunicación médico-paciente en los oncólogos del sur de Europa: la influencia de la orientación psicosocial y el agotamiento. RET Rev Toxicom 2005;44:15-24.

94. Barbero J, Barreto, P, Arranz P, Bayés R. Importancia de la comunicación en oncología. Comunicación en oncología clínica. Madrid: Just in Time SL; 2005.

95. Merckaert I, Libert Y, Delvaux N, Marchal S, Boniver J, Etienne AM, et al. Factors that influence physicians' detection of distress in patients with cancer: Can a communication skills training program improve physicians' detection? Cancer 2005;104(2):41121.

96. Mercier M, Schraub S, Bransfield D, Barthod L. Mesure de la qualite de vie. Application au dépistage de la souffrance psychologique chez les patients cancereux. Bull Cancer 1992;79(2):193-204.

97. Worden JW. Psychosocial screening of cancer patients. J Psychosoc Oncol 1983;1(4):1-10.

98. Holland JC; Reznik I. Pathways for psychosocial care of cancer survivors. Cancer 2005;104, 2624-37. Doi: 10.1002/ cncr.21252.

99. Middleboe T, Ovesen L, Mortensen EL, Bech P. Depressive symptoms in cancer patients undergoing chemotherapy: A psy- 
chometric analysis. Psychother Psychosom 1994;61(3-4):171-7.

100. Pruitt BT, Waligora-Serafin B, McMahon T, Davenport J. Prediction of distress in the first six months after a cancer diagnosis. J Psychosoc Oncol 1992; 9(4):91-12.

101. Zabora JR, Smith-Wilson R, Fetting JH, Enterline JP. An efficient method for the psychosocial screening of cancer patients. Psychosomatics 1990;31:192-6.

102. Razavi D, Delvaux N, Farvaeques C, Robaye E. Screening for adjustment disorders and mayor depression disorders in cancer inpatients. Br J Psychiatry 1990;156:79-83.

103. Silverstone PH. Poor efficacy of the Hospital anxiety and Depression Scale in both medical and psychiatric patients. J Psychosom Res 1994;38(5):441-50.

104. Tejero A, Guimerá EM, Farré JM, Peri JM. Uso clínico del HAD (Hospital Anxiety and Depresión Scale) en población psiquátrica: un estudio de sensibilidad, fiabilidad y validez. Rev Dept Psiquiatría Fac Med Barna 1986;13:233-8.

105. Lewis G, Wessely S. Comparison of the General Health Questionnaire and the Hospital Anxiety and Depression Scale. Br J Psychiatry 1990;157:860-4.

106. Herrmann C. International experiences with the Hospital Anxiety and Depression Scale: a review of validation data and clinical results. J Psychosom Res 1997;42:17-41.

107. Hopwood P, Howell A, Maguire P. Screening for psychiatric morbidity in patients with advanced breast cancer: validation of two serl-report questionnaires. Br J Cancer 1991;64:353-6.

108. Le Fevre P, Devereux J, Smith S, Lawrie SM, Cornbleet M. Screening for psychiatric illness in the palliative care inpatient setting: A comparison between the Hospital Anxiety and Depression Scale and the General Health Questionnaire-12. Palliat Med 1999;13:399-407.

109. López-Roig S, Terol MC, Neipp MC, Leyda I, Massutí B, Picó C, et al. Assessing anxiety and depression with HADS scale in a Spanish cancer population: A preliminary validation study. Psychoooncology 1998;7:109-16.

110. Costa G, Perez X, Salamero M, Gil FL. Discriminación del malestar emocional en pacientes oncológicos utilizando la escala de ansiedad y depresión hospitalaria (HADS) Ansiedad Estrés. 2009;15(23):217-29.

111. Braeken AP, Lechner L, Gils FC, van Houben RM, Eekers D, Ambergen T, et al. The effectiveness of the screening inventory of psychosocial problems (SIPP) in cancer patients treated with radiotherapy: design of a cluster randomised controlled trial. BMC Cancer 2009;9:177.

112. Derogatis LR. The brief sympton inventory administration, scoring, and procedures Manual-II. New York: Clinical Psichometric Research; 1992.

113. Stefanek ME, Derogatis LP, Shaw A. Psychological distress among oncology outpatients. Prevalence and severity as measured with the Brief Symptom Inventory. Psychosomatic 1987;28(10):530-8.

114. Derogatis LR. Brief symptom inventory (BSI). 18 administration, scoring, and procedures manual. Minneapolis, MS: National Computer Systems; 2000.

115. Morasso G, Costantini M, Baracco G, Borreani C, Capelli M. Assessing psychological distress in cancer patients: validation of a self-administered questionnaire. Oncology 1996;53:295-302. Doi: $10.1159 / 000227576$.

116. Spielberger CD. Manual for the statetrait anxiety inventory (form Y). Palo Alto, California: Consulting Psychologists Press; 1983.

117. Bruera E, Kuehn N, Miller MJ, Selmser P, Macnillan K. The Edmonton symptom assessment system: a simple method for the assessment of palliative care. J Palliat Care 1991;7:6-9.

118. Butt Z, Wagner LI, Beaumont JL, Paice JA, Peterman AH, Shevrin D, et al. Use of a single-item screening tool to detect clini- 
cally significant fatigue, pain, distress, and anorexia in ambulatory cancer practice. J Pain Symptom Manage 2008;35:20-30. Doi:10.1016/j.jpainsymman. 2007.02.040.

119. Ordoñez A, González M. La astenia tumoral: un síndrome poco estudiado. Psicooncología. 2004;2-3(1):25-8.

120. Mitchell AJ. Pooled results from 38 analyses of the accuracy of distress thermometer and other ultra-short methods of detecting cancer-related mood disorder. J Clin Oncol 2007;25:4670-81.

121. Mitchell AJ, Coyne JC. Do ultra-short screening instruments accurately detect depression in primary care?: A pooled analysis and meta-analysis of 22 studies. Br J Gen Pract 2007;57:144-51.

122. Trask PC, Paterson A, Riba M, Brines B, Griffith K, Parker P, et al. Assessment of psychological distress in prospective bone marrow transplant patients. Bone Marrow Transplant. 2002;29(11):917-25.

123. Akizuki N, Akechi T, Nakanishi T, Yoshikawa E, Okamura M, Nakao T, et al. Development of a brief screening interview for adjustment disorders and major depression in patients with cancer. Cancer 2003;97(10):2605-13.

124. Jacobsen PB. Screening for Psychological Distress in Cancer Patients: ChaIlenges and Opportunities. J Clin Oncol 2007;25(29):4526-27.

125. Cohen M, Gagin R, Cinamon T, Stein T, Moscovitz M, Kuten A. Translating 'distress' and screening for emotional distress in multicultural cancer patients in Israel. Qual Life Res 2012;21:555-562.

126. Ziegler L, Hill K, Neilly L, Bennett MI, Higginson IJ, Murray SA, Stark D. Identifying psychological distress at key stages of the cancer illness trajectory: A systematic review of validated self-report measures. J Pain Symptom Manage 2011;41(3):619-36.

127. Chochinov HM, Wilson KG, Enns M, Lander S. "Are You Depressed?" Screening for depression in the terminally ill. Am J Psychiatry 1997;154(5):674-6.
128. Spitzer RL, Endicott J, Robins E, Research Diagnostic Criteria: rationale and reliability. Arch Gen Psychiatry 1978;35:773-82.

129. Endicott J, Spitzer RL. A diagnostic interview: the schedule for affective disorders and schizophrenia. Arch Gen Psychiatry 1978;35:837-44.

130. Beck AT, Beck RW. Screening depressed patients in family practice: A rapid technique. Postgrad Med 1972;52:81-5.

131. Volk RJ, Pace TM, Parchman ML. Screening for depression in primary care patients: Dimensionality of the short form of the Beck Depression Inventory. Psychol Assess 1993;5:173-81.

132. Davey HM, Barratt AL, Butow PN, Deeks JJ. A one-item question with a Likert or Visual Analog Scale adequately measured current anxiety. J Clin Epidemiol 2007;60:356-60.

133. Bayés R, Limonero JT, Barreto $P$, Comas MD. A way to screen for suffering in palliative care. J Palliat Care 1997;13(2):22-6.

134. Maté J, Mateo D, Bayés R, Bernaus $M$, Casas C, González-Barboteo J, et al. Elaboración y propuesta de un instrumento para la detección de malestar emocional en enfermos al final de la vida. Psicooncología 2009;6(2-3):507-18.

135. Limonero JT, Mateo D, Maté-Mendez J, González-Barboteo J, Bayés R, Bernaus M, Casas C, López M, Sirgo A, Viel S. Evaluación de las propiedades psicométricas del cuestionario de Detección de Malestar Emocional (DME) en pacientes oncológicos. Gac Sanit 2012;26:145-52. Doi:10.1016/j.gaceta.2011.07.016.

136. Sheehan DV, Lecrubier Y, Harnett-Sheehan $\mathrm{K}$, Amorim P, Janavs J, Weiller $\mathrm{E}$, et al. The MINI International Neuropsychiatric Interview (MINI): The development and validation of a structured diagnostic psychiatric interview. J Clin Psychiaty 1998;59(20):22-23. Doi:10.1016/S09249338(99)80239-9.

137. Ferrando L, Bobes J, Gibert J, Soto M, Soto O. MINI. Entrevista Neuropsiquiátri- 
ca Internacional. Versión en Español 5.0.0. DSM-IV. Traducida por L. Franco-Alfonso, L. Franco. 2000 [Bajada 2005]. Disponible en: http://entomologia.rediris.es/pub/bscw. cgi/d602335/ MINI/EntrevistaNeuropsiquiatritalnternacional.pdf.

138. Coyne JC, Benazon NR, Gaba CG, Calzone K, Weber BL. Distress and psychiatric morbidity among women from high-risk breast and ovarian cancer families. J Consult Clin Psychol 2000;68(5):864-74. Doi: 10.1037/0022-006X.68.5.864.

139. Sandanger I, Moum T, Ingebrigtsen G, Dalgard OS, Sørensen T, Bruusgaard D. Concordance between symptom screening and diagnostic procedure: the Hopkins Symptom Checklist-25 and the Composite International Diagnostic Interview I. Soc Psychiatry Psychiatr Epidemiol 1998;33:34554. Doi:10.1007/s001270050064.

140. Kirsh KL, McGrew JH, Dugan M, Passik SD. Difficulties in screening for adjustment disorder. Part I: Use of existing screening instruments in cancer patients undergoing bone marrow transplantation. Palliat Support Care 2004;2(1):23-31. Doi:10.1017/ S1478951504040040.

141. Kawase E, Karasawa K, Shimotsu S, Imasato $\mathrm{S}$, Ito $\mathrm{K}$, Matsuki $\mathrm{H}$, et al.
Evaluation of a one-question interview for depression in a radiation oncology department in Japan. Gen Hosp Psychiatry 2006;28:321-2. Doi:10.1016/j. genhosppsych.2006.02.003.

142. Akechi T, Okuyama T, Sugawara Y, Shima Y, Furukawa TA, Uchitomi Y. Screening for depression in terminally ill cancer patients in Japan. J Pain Symptom Manage 2006;31(1):5-12.

143. Lloyd-Williams M, Dennis M, Taylor F. A prospective study to compare three depression screening tools in patients who are terminally ill. Gen Hosp Psychiatry 2004;26(59):384-9. Doi:10.1016/j.genhosppsych.2004.04.002.

144. Lloyd-Williams M, Dennis M, Taylor F, Baker I. Is asking patients in palliative care -are you depressed- appropriate? Prospective study. BMJ 2003;327:372-3. Doi:10.1136/bmj.327.7411.372.

145. Vodermaier A, Linder W, Siu C. Screening for emotional distress in cancer patients: a systematic review of assessment instruments. JNCI. 2009;101:1464-88. Doi:10.1093/jnci/djp336.

146. Mitchell A. Short detection tools for cancer-related distress: A review and diagnostic validity meta-analysis. JNCCN 2010;8:487-94. 
